\title{
Visualizing the multiscale structural dynamics of photoexcited molecular complexes with ultrafast hard X-rays
}

\section{Sophie Canton}

\author{
European XFEL, Schenefeld, Germany; \\ sophie.canton@xfel.eu
}

\begin{abstract}
Visualizing on the atomic scale the full extent of the electronic and structural changes that are triggered by charge separation and subsequent charge transport is crucial for developing the rational design of novel sensitizers and catalysts. The rapid progress of ultrafast X-ray techniques, both at synchrotrons $(100 \mathrm{ps})$ and at X-ray free electron laser facilities (sub-ps) have equipped the scientific community with novel analytical tools that are capable of delivering unique feedback with spin and elemental sensitivity about the highly-correlated nonadiabatic dynamics that follow photoabsorption. The present talk will review the technical state-of-the art and the ongoing developments that are currently taking place. The talk will also highlight several of the recent results that have been obtained for intramolecular and interfacial processes of relevance for the function and optimization of advanced materials.
\end{abstract}

Keywords: time-resolved XAS and WAXS, large scale facilities 